\title{
Application of Computer Software Technology in Plant Protection Software Development
}

\author{
Yejia Shi, a \\ ${ }^{1}$ Liaoning Railway Vocational and Technical College, Jinzhou, Liaoning, China, 121000 \\ ${ }^{a}$ email,
}

Keywords: Application, Computer Software Technology, Plant Protection Software Development

\begin{abstract}
With the continuous development of computer software technology, in order to develop the plant protection software which is more practical and more complete, people began to use computer software technology in the development process of plant protection software. In this paper, from the role of plant protection software, the application of computer software technology in the plant protection software development is analyzed.
\end{abstract}

\section{Introduction}

At present, with the rapid development of information technology in China, the acceleration of network construction in some areas has provided a good opportunity for the improvement of plant protection computer application level. Plant protection departments all over the country have been or are preparing to configure higher-level computer hardware equipment, and the corresponding lack of plant protection computer application software has become a constraint to improve the level of plant protection computer applications. Therefore, the development of high level of plant protection computer application software is imminent, but also to improve the level of application of computer protection plant protection must be the way.

Computer with its high-speed, intelligent outstanding features have gradually penetrated into the human society, economy, culture, life in many ways. All walks of life are using computer technology to promote its modernization and scientific process. From a global perspective, the application of computers in agriculture has been more than 40 years of history, has experienced four major stages (50-60 years of scientific computing period, 70 years of data processing time, 80 years of knowledge processing period). On the application of computer agriculture, began in the late $70 \mathrm{~s}$ and early 80s, started late. However, due to the great importance of the country and the unremitting efforts of the majority of agricultural science and technology workers, its rapid development, is now from the initial stage to a multi-level, large-scale direction. Plant protection application software development, after 10 years of accumulation, especially in the past three years of rapid development, has become a computer application of agriculture in a more active field, and in agricultural production and pest management plays an important role. So, how to more effectively carry out high-quality plant protection application software development? Software engineering theory and method of flexible use is the key to solve this problem.

\section{The Basic Concept of Software Engineering}

With the development of computer applications in the 1960s, the number and size of software are growing. During this period the cost of the software has risen sharply but its reliability has declined, which is commonly referred to as the software crisis. It is in this context that people begin to notice that the production of software should also be seen as a project, that is, the engineering approach should be introduced into the software development. In 1968, the concept of "software engineering" was presented at a meeting of the North Atlantic Treaty Group on the subject of the software crisis. Software engineering is simply to develop and use complete engineering regulations, good management strategies, the development of the use of appropriate tools and methods, in the appropriate resources under the conditions of the appropriate equipment, access to high-quality software with clear meaning. 
In the past 30 years on the basis of progressive development, software engineering has formed a more mature theory and methods. According to the general principles of software engineering, a software development from the plan to the abandoned not known as the software's life, usually including planning, development and operation of the period. In accordance with its principles, the survival of the various periods can be subdivided into several smaller stages, different stages of the division method, constitute a different model of software survival, also known as software development model. In the development of software engineering, there have been different types of software development models, such as waterfall model, rapid prototyping model, operating model and software evolution model. In many engineering software development methods, the waterfall model with its distinctive features and effective way to become the most mature and popular model, especially for small and medium-sized software development. Plant protection application software is generally medium-sized software, many years of practice proved that the waterfall model is the development of high-quality plant protection software, an effective method.

\section{The Application of Computer Software Technology in Plant Protection Software Development}

In the current situation, the science and technology personnel in the plant protection disciplines should take the software engineering theory as the guide, grasp the development trend of computer application, select suitable software development methods and software development tools for the plant protection professional and technical personnel, organize the software developers, High-level, high-quality plant protection application software system.

Use Software Engineering Theory As the Guide. According to the general principles of software engineering, a software design from the plan to the end of the software is not known as the survival, can be divided into six phases: software planning - software requirements analysis software design - software coding - software testing - software maintenance, survival The various stages of the period can be broken down into several smaller stages, different stages of the division method to form a different software model of survival, also known as software development model. In the development of software engineering, many kinds of development models have been developed, such as waterfall model and rapid prototyping model. It is worth noting that the different software development methods have different characteristics, the emphasis on the software life stage of the project is not the same degree of emphasis, but the software life cycle is still a software engineering view of the basic point, reflecting the software, Development should follow the inherent law. The development of plant protection application software system should be guided by the principle and method of software engineering. Software engineering theory and method are the basic guarantee for the successful development of high quality plant protection software. Because: ${ }^{1}$ can effectively carry out system planning and design, detours, to avoid undue errors, thus shortening the development cycle, saving manpower and material resources. In order to successfully carry out system development, quality assurance is the premise and provides users with correct and reliable application software. Can carry on the system maintenance scientifically keep the exuberant vitality of the software in the practical application. Review In recent years has developed a more excellent plant protection application software, such as the Green Cross Doctor System (National Agricultural Technology Extension Service Center), the armyworm migration expert system (Chinese Academy of Agricultural Sciences Institute of Plant Protection), pear black star Disease prediction and management expert system ESPSPM, pest information system, plant virus quarantine diagnosis and support system PQV-DSS and the northern region of vegetable pest identification system PESTDIAG, etc., the development of these software are all infiltrated the idea of software engineering. The wheat aphid forecast system in Huang-Huaihai area was designed according to the software engineering theory, and the rapid prototyping method was used to develop the system. .

The Choice of Development Platform. The operating system is one of the most important computer system software. Operating system is the computer system's own hardware and software resources for comprehensive control and management (storage management, processor management, equipment management, information management and job management) procedures, so that the 
computer under the command of its normal operation, all other software installed on the computer relies on the instructions of the operating system to complete the work. Operating system is the user and computer interface, which is the application software development platform. When choosing the development platform, we should give full consideration to the practicality, simplicity, maintainability, scalability, cost performance, applicability to the network, the popularity of the basic support environment and the future development direction of the computer application. At present, the Windows system because of user-friendly, easy to operate and so much the user's welcome, therefore, Windows-based application software has become today's software developers and users of the preferred program. Taking the development of HH-AphidGIS in Huang-Huai-Hai area as an example, Windows 3.1 was selected as the platform of system development. But the development direction of Microsoft 32-bit Windows 95 operating system has gradually become the main platform for computer applications, and Windows 95/98 / NT as the future of computer and network systems, the main application platform, the momentum is unstoppable; At the same time, the computer application of each regional forecasting department is gradually transplanted to Windows 95/98 platform, and Windows 95/98 will be the main platform for the future application of the system. So HH-AphidGIS system finally gave up Windows3.1, choose Windows 95 as the development platform, in order to ensure its advanced technology, and consistent with the future development direction of computer application.

The Choice of Development Methods. According to the software engineering theory, it is very important to choose the development method which is suitable for the system requirement after analyzing and designing the system. In general, for the application software system development approach can be summarized into two kinds: the system developers using Borland $\mathrm{C}++$, VisualBASIC or Delphi and other languages directly programming, known as the direct programming method. This method development cycle is long, high cost, after the completion of the system stability and robustness required a lot of work to ensure that, and the requirements of the developer. However, this method has the advantage of flexibility, the developer can give full play to their imagination and flexibility to design a variety of user interface changes and to fully implement the system requires a variety of special features.

The use of existing market development tools, such as Authorware, Multibase, etc. to achieve, known as the tool method. This method short development cycle, low development costs, developers generally do not need programming, by writing scripts, writing description language or editing cards to complete the application system. The robustness and stability of the system depends on the development platform or development system used. Developers are difficult to break through the development platform provides a variety of functional limitations, and thus the development of the application system is also more modular.

For a specific system, according to the needs of the system design phase analysis and the development of the actual situation to choose, such as VegePest as a multimedia database is to choose the tool method as the development method of its system, and PestDiag as a multimedia expert system, The need for rigorous reasoning, some of the current software tools do not have this ability, which chose the direct programming method. HH-AphidGIS system is a practical system integrating geographic information system, multimedia, database management, forecasting and forecasting. From the demand analysis of system development, HH-AphidGIS system not only needs the support of multimedia function, but also mainly Therefore, the first method, the direct programming method, has been chosen for the development of wheat aphid, which needs to be modeled and the interaction between model and GIS cannot be satisfied by the tool method.

The Selection of Development Tools. If you select the application of direct programming method for plant protection application software system development, select the appropriate development tools to become a serious consideration must be an important issue. How to choose the right tools depends on the operating system platform you are using, the ease of use of the development tools, the scope of the application, the issues to be addressed, the scale, the direction of technology development, and scalability. The choice of tools is necessary to meet the functional requirements of the application system, but also to meet their performance needs (such as 
computing speed, print speed, etc.), can also be mixed in several different language platform programming in order to learn from each other. At present the development of plant protection application software should be a visual tool for the development of tools of choice.

With the continuous development of computer technology, programming tools have also been rapid development. The introduction of Visual BASIC makes it possible to "write a Windows application in minutes" is no longer a myth. The introduction of Borland Delphi 2.0 is to make visual programming tools gradually into the boom. Especially since 1997, visual programming tools become a very active area, the software vendors have introduced their new version of the product, in terms of functionality, performance, etc. have greatly improved and the competition between the products is also more intense.

The Composition of the Developer. The composition of computer application software system developers is changing with the development of programming language and development tools. In particular, in recent years the development of object-oriented theory and the development of visual tools, greatly reducing the difficulty of application software development, reducing the amount of writing code, improve the efficiency of software development for the majority of non-computer Professional workers (including plant protection workers) have developed a high-quality application software system in the field to provide a reliable tool to enable the development of application software systems in various fields to get rid of the limitations of computer professionals, and experts in various fields And gradually become the leading application software system development.

Based on the above analysis and from the IPMIST laboratory more than 10 years of practical experience, the current plant protection application software system development can plant protection professionals. Of course, some of them should be both knowledge of software engineering theory and programming knowledge, but also experts in the field of plant protection. From application software system analysis, design, to the final realization of the system, system developers should focus on how to reflect the characteristics of plant protection professionals, plant protection expertise in the collection, collation and its connotation, such as in-depth excavation, and Not to focus on the preparation of lengthy tedious lines of code. Today, the development of software technology, especially the development of object-oriented technology and visualization tools to provide a convenient condition, so the system designers and application experts can be organically integrated into one.

\section{Conclusion}

The application of computer software technology to the development of plant protection software effectively promoted the development efficiency and quality of the software. In this process, the theory of software engineering should be taken as the guidance of plant protection software development, and the selection of suitable development platform, development method and development tool should be paid attention to. The development of tools and programming languages has enabled more experts in the field of plant protection to participate in the development process of plant protection software. The participation of experts has promoted the development of plant protection software.

\section{References}

[1] Jia Xinzhang, Li Jingyuan. Agricultural Engineering, Vol. 6 (2014) No 53, p.25-26

[2] Peng Sue, Wang Yunhui, Wang Qunyong. Microcomputer, Vol. 12 (2015) No 27, p.74-76

[3] Qian Xiyuan, Jing Jianfen. Journal of Shihezi University, Vol. 30 (2014) No 19, p.144-145

[4] Wang Kuailiang. Plant Protection, Vol. 29 (2008) No 27, p.21-23

[5] Zhang Gongxu, Sun Jing. Pesticide Market Information, Vol. 8 (2013) No 27, p.57-60 